\title{
Roughness correction to the Casimir force beyond perturbation theory
}

\author{
Wijnand Broer ${ }^{1}$, George Palasantzas ${ }^{1}$, Jasper Knoester ${ }^{1}$, and Vitaly B. Svetovoy ${ }^{2}$ \\ Zernike Institute for Advanced Materials, University of Groningen, Nijenborgh 4, 9747 AG Groningen, The Nether- \\ lands \\ $\mathrm{MESA}^{+}$Institute for Nanotechnology, University of Twente, P.O. Box 217, 7500 AE Enschede, The Netherlands
}

PACS $03.70 .+\mathrm{k}-$ Theory of quantized fields

PACS 68.37.Ps - Atomic force microscopy (AFM)

PACS 85.85.+j - Micro- and nano-electromechanical systems (MEMS/NEMS) and devices

\begin{abstract}
Up to now there has been no reliable method to calculate the Casimir force when surface roughness becomes comparable with the separation between bodies. Statistical analysis of rough $\mathrm{Au}$ films demonstrates rare peaks with heights considerably larger than the root-meansquare (rms) roughness. These peaks define the minimal distance between rough surfaces and can be described with extreme value statistics. We show that the contributions of high peaks to the force can be calculated independently of each other while the contribution of normal roughness can be evaluated perturbatively beyond the proximity force approximation. The developed method allows a reliable force estimation for short separations. Our model explains the strong hitherto unexplained deviation from the normal Casimir scaling observed experimentally at short separations.
\end{abstract}

Introduction. - The Casimir force 1 attracts increasing attention nowadays since modern technology allows dimension control at distances $\leq 100 \mathrm{~nm}$ where this force becomes operative (see [2,3] for a review). Indeed, modern micro/nano-electromechanical (MEM/NEM) engineering is now being conducted at the micron to nanometer scale and has attracted interest in the Casimir force 4. MEM devices such as vibration sensors and switches are now routinely made with parts a few micrometers in size, and have the right size for the Casimir force to play a role. This is because MEM systems have surface areas large enough but gaps small enough, for the force to draw components together and possibly lock them permanently _-' an effect known as stiction. Such permanent adhesion (in addition to capillary adhesion due to the water layer) is a common cause of malfunctioning of MEM devices [5] [7].

In this range of separations the force appears mainly due to quantum fluctuations of the electromagnetic field (zero-point field) in the interacting bodies while at larger distances classical (thermal) fluctuations become increasingly important 8, 9 . The famous Casimir formula $F_{C}=$ $\left(\pi^{2} / 240\right)\left(\hbar c / d^{4}\right)$ gives the force (per unit area) at temperature $T=0$ between two ideally reflecting semi-spaces separated by the distance $d$. The force measured in recent experiments (see 3 for a review) can deviate significantly from the ideal case because the temperature is finite, the bodies are not ideal reflectors, and the distance between them is not well defined. Considerable efforts were made to improve the Casimir formula. Indeed, the more detailed description is based on the Lifshitz formula, which accounts for actual optical properties of interacting bodies and nonzero temperature. The optical data were included in the calculational procedure [10,11. Although the thermal correction to the Casimir force is rather controversial [12, 13, it is not important for the short distances discussed in this paper.

An important correction to the Casimir force that is not accounted for by the Lifshitz formula is the roughness correction. The surfaces of real bodies are rough, which makes the distance between them not well defined. The first attempts to account for roughness [14] were based on the proximity force approximation (PFA). In this approximation the real surfaces are replaced by flat patches and the force was calculated as the sum of forces between opposite patches, treating such pairs as parallel plates. For the dispersive forces the PFA was applied for the first time by Derjaguin [15.16]. The approximation is justified when the separation $d$ is much smaller than the local curvature 
radius and size of patches. It is well suited for smooth large bodies, but works worse for roughness corrections. It was noted [17] that in order to apply the PFA to rough bodies the roughness correlation length $\xi$ (typical features size on the surface) must be larger than the separation, $\xi \gg d$. Then the result found in 14 will be true for small root mean square (rms) roughness, $w \ll d$. However, in most of the experimental situations the condition $\xi \gg d$ is broken and more elaborate theory has to be used to calculate the roughness correction. This theory was developed in refs. 18, 19. It treats the roughness contribution through second order perturbation theory in $w / d$. The theory showed larger corrections than those predicted within the PFA. In fact, the correction is very important at short separations and has to be carefully included for interpretation of the force experiments exploring short distance ranges.

The Casimir forces between a gold covered sphere and plates of different roughness were measured for separations from 20 to $200 \mathrm{~nm}$ [20]. The films with larger rms roughness at short separations demonstrate significant (more than 100\%) deviations from the theoretical expectations based on the perturbative roughness correction. Empirically it was established that the minimal distance between two rough bodies (distance upon contact) is $d_{0} \approx 3.7\left(w+w_{s p h}\right)$, where $w_{\text {sph }}$ is the sphere's rms roughness. Because $d_{0}$ is the minimum separation distance, the perturbative correction must be smaller than $K\left(w+w_{\text {sph }}\right)^{2} / d_{0}^{2}=0.07 K$, where $K \sim 10$ is a large numerical factor (due to sharp behavior of the force with the distance). It was concluded [20] that at short separations the perturbation theory fails. These experimental results still did not get a theoretical explanation. Moreover, we are facing a problem: there is no reliable method to estimate the roughness correction when $d$ becomes comparable with the rms roughness $w$. In this paper we propose a method to address this problem by combining the PFA and perturbation theory approaches. Although we will prove the applicability of this method specifically for gold films, we believe that similar approaches can be developed for other materials, after detailed analysis of the roughness statistics obtained, e.g., in terms of scanning probe microscopy techniques.

Statistics of rough surfaces. - The distance upon contact $d_{0}$ was discussed in detail for gold films 21. The films deposited with different thicknesses have different rms roughnesses due to kinetic roughening processes. For all these films atomic force microscope (AFM) images were recorded for large area (of up to $40 \times 40 \mu \mathrm{m}^{2}$ ) with lateral resolution of 4-10 nm. This information allows a detailed analysis of the roughness statistics. The probability to find a height of a local feature smaller than some value $z$ can be presented in a general form

$$
P(z)=1-e^{-\phi(z)},
$$

where for convenience we introduced the "phase" $\phi(z)$ as nonnegative and nondecreasing function of $z$. The phase describes the roughness distribution in a convenient way, which makes it possible to calculate the contributions from peaks and troughs as will be shown later. It was already noted 21] that the cumulative distribution $P(z)$ for gold films cannot be described satisfactorily by any known distribution at all $z$ but asymptotically at large $z$ it can be fitted with generalized extreme value distributions 22 .

We performed a special analysis of the AFM surface data presented in ref. 21] to reveal the best asymptotic distribution at large $|z|$. In this limit the phase $\phi(z)$ is much more convenient for analysis than $P(z)$. This is because $P(z)$ approaches very fast 0 or 1 in the limit $|z| \rightarrow$ $\infty$. Indeed, we can present the phase as

$$
\phi(z)=-\ln [1-P(z)]
$$

where $P(z)$ is extracted directly from the images. The function $\phi(z)$ for an $1600 \mathrm{~nm}$ thick gold film is shown in fig. 11 The inset shows the probability density function $f(z)=d P / d z=(1-P) d \phi / d z$. Similar behavior is realized for all investigated gold films. It is clear that for large positive $z$ the logarithm of the phase can be fitted with a linear function

$$
\ln \phi(z)=A+B z, \quad z \rightarrow \infty
$$

and similarly for large negative $z$. With this $\phi$ the probability to find a feature larger than $z$ behaves asymptotically as a double exponential

$$
1-P(z) \sim \exp \left[-\exp \left(\frac{z-\mu}{\beta}\right)\right],
$$

where $\beta$ and $\mu$ are the scale and location parameters respectively. This behavior is a characteristic feature of the Gumbel distribution [23, which is an example of extreme value statistics. In this paper only gold films were analyzed and therefore we cannot draw conclusions on the roughness statistics of other materials. However, the extreme character of the statistics allows us to hope that this behavior is more general.

Roughness correction to the Casimir force. We can imagine a rough surface as a large number of asperities with heights $\sim w$ and lateral size $\xi$, and occasional high peaks and deep troughs. These peaks (or troughs) are high in the sense that their height is considerably larger than $w$, say $>3 w$. The situation can be visualized as a lawn covered with grass and occasional high trees standing here and there. In this paper we propose a method to calculate the roughness correction to the Casimir force on the basis of this separation. Namely, the asperities with the height $\sim w$ can be taken into account using perturbation theory, without the use of the proximity force approximation. On the other hand, for high peaks the local distance between interacting bodies becomes considerably smaller and one cannot use perturbation theory anymore. Because high peaks are rare the average distance $l$ between them is large. If this distance is so large that $l \gg d$, then we can 


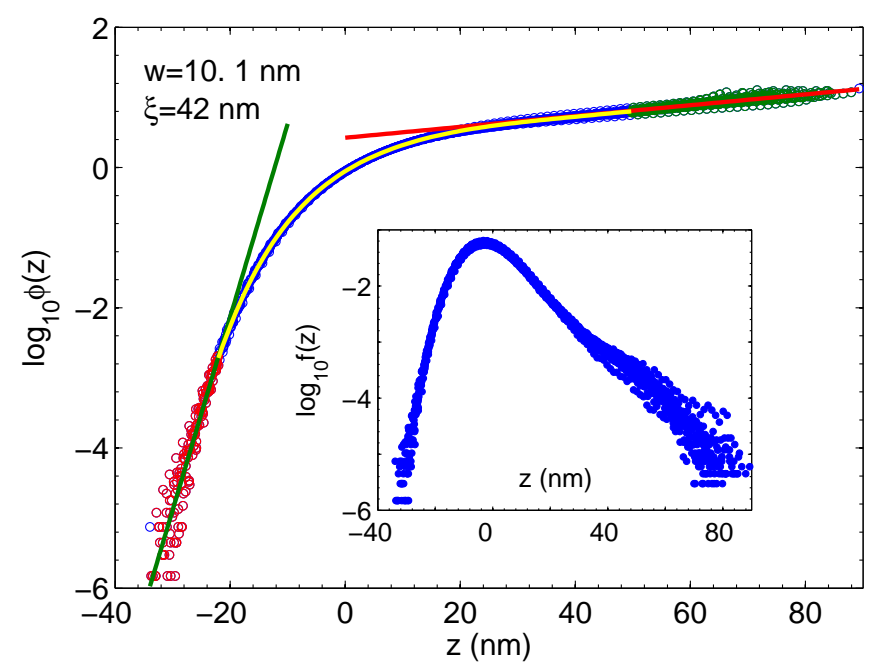

Fig. 1: (Color online) The "phase" as a function of $z$ for a 1600 $\mathrm{nm}$ gold film. The open circles are the actual data extracted from the AFM image using eq. (2). At large positive and large negative heights $\log _{10} \phi(z)$ is well fitted by linear functions of $z$ as is shown by the straight lines. The curved line is a polynomial fit at intermediate $z$. The inset shows the probability density function $f(z)$. It demonstrates significant deviation from a normal distribution.

calculate the contribution of these peaks independently of each other, as it is assumed in the PFA. However, this contribution has to be calculated beyond the perturbation theory. It has to be stressed that the interaction of a separate peak with a flat surface can be taken into account precisely using developed numerical or analytical methods 3.

The number of asperities $N$ with the height $d_{1}>3 w$ and lateral size $\xi$ on the area $L^{2}$ is given by the equation $21]$

$$
N=\frac{L^{2}}{\xi^{2}} e^{-\phi\left(d_{1}\right)}
$$

The average distance between these peaks is

$$
l=\frac{L}{\sqrt{N}}=\xi e^{\phi\left(d_{1}\right) / 2} .
$$

In order to fulfill the condition of PFA applicability $l \gg d$, we can choose the parameter $d_{1}$ from the interval $3 w<$ $d_{1}<d_{0}$, where $d_{0}$ is the maximal peak on the area $L^{2}$. The best choice for $d_{1}$ will be discussed later. Similarly, one can introduce the average distance $l^{\prime}$ between deep troughs

$$
l^{\prime}=\frac{L}{\sqrt{N^{\prime}}}=\frac{\xi}{\sqrt{\phi\left(-d_{1}^{\prime}\right)}},
$$

where $d_{1}^{\prime}$ has to be chosen from the interval $3 w<d_{1}^{\prime}<d_{0}^{\prime}$ to fulfill the condition $l^{\prime} \gg d$ and $d_{0}^{\prime}$ is the deepest trough on the area $L^{2}$.

Here we consider the general case where we are interested in the Casimir force between two plates with rough surfaces. As was explained in ref. 21] this is equivalent to the interaction of a smooth plate with a rough one, which has the combined roughness topography $h(x, y)=h_{1}(x, y)+h_{2}(x, y)$, where $h_{1,2}(x, y)$ are the topographies of the interacting plates 1 or 2 . Therefore all the equations above have to be applied to the combined roughness profile $h(x, y)$.

Let us assume for a moment that the PFA can be applied to any roughness topography. Then the force between the plates can be calculated using the standard definition of the averaged function

$$
\mathcal{F}(d)=\int_{d_{1}}^{d_{0}} \ldots+\int_{-d_{0}^{\prime}}^{-d_{1}^{\prime}} \ldots+\int_{-d_{1}^{\prime}}^{d_{1}} d z f(z) F(d-z)
$$

where $f(z)$ is the probability density function, and we separated high peaks (first integral), deep troughs (second integral), and the normal roughness contribution (third integral). For the moment we do not specify the force between the interacting patches $F(d)$ separated by the distance $d$. The last term can be calculated using the perturbation expansion $F(d-z)=F(d)-F^{\prime}(d) z+F^{\prime \prime}(d) z^{2} / 2 !+\ldots$ and we find for this term

$$
\int_{-d_{1}^{\prime}}^{d_{1}} \ldots=F(d) \int_{-d_{1}^{\prime}}^{d_{1}} d z f(z)+\frac{F^{\prime \prime}(d)}{2 !} \int_{-d_{1}^{\prime}}^{d_{1}} d z f(z) z^{2} .
$$

The first and second integral on the right are 1 and $w^{2}$, respectively, if one extends the integration limits to infinity. When the applicability of the PFA breaks down the second term in (9) (with infinite limits) can be generalized as follows 17

$$
\mathcal{F}_{P T}(d)=\frac{F^{\prime \prime}(d)}{2 !} \int \frac{d^{2} k}{(2 \pi)^{2}} \rho(k d) \sigma(k) .
$$

Here $\sigma(k)$ is the Fourier spectrum of the roughness correlation function. The function $\rho(k d)$ measures the deviation from the PFA. When the PFA is applicable this function is $\rho(k d)=1$ and we reproduce eq. (9). Outside of the PFA applicability we can use for $\rho(k d)$ expressions found in 18, 19.

The term $\mathcal{F}_{P T}(d)$ is the roughness contribution to the force treated as a perturbation theory correction. As we know already the contribution of high peaks (or deep troughs) may not be accounted for by the perturbation theory. However, in this case we can account for the peaks (troughs) independently and the contribution can be presented as the first (second) term in (8). Taking into account the change of the integration limits we find the contributions due to high peaks, $\mathcal{F}_{P F A}(d)$, and due to deep troughs, $\mathcal{F}_{P F A}^{\prime}(d)$,

$$
\begin{aligned}
& \mathcal{F}_{P F A}(d)= \\
& \quad \int_{d_{1}}^{d_{0}} d z f(z)\left[F(d-z)-F(d)+F^{\prime}(d) z-\frac{F^{\prime \prime}(d)}{2 !} z^{2}\right],
\end{aligned}
$$




$$
\begin{aligned}
& \mathcal{F}_{P F A}^{\prime}(d)= \\
& \quad \int_{-d_{0}^{\prime}}^{-d_{1}^{\prime}} d z f(z)\left[F(d-z)-F(d)+F^{\prime}(d) z-\frac{F^{\prime \prime}(d)}{2 !} z^{2}\right]
\end{aligned}
$$

The final expression for the force that includes the total roughness contribution can be presented as

$$
\mathcal{F}(d)=F(d)+\mathcal{F}_{P T}(d)+\mathcal{F}_{P F A}(d)+\mathcal{F}_{P F A}^{\prime}(d) .
$$

Here $F(d)$ is the force between flat surfaces and the other three terms are the different roughness corrections.

The same force $F(d)$ is used to calculate $\mathcal{F}_{P F A}(d)$ and $\mathcal{F}_{P F A}^{\prime}(d)$, which implies that high peaks are described as pillars with flat faces. However, this approximation is not necessary. If the peaks can be considered as independent, then the interaction of each peak with the flat surface can be described precisely (numerically) or approximately with an appropriate force $\tilde{F}(d)$ in eqs. (11) and (12), taking into account the actual geometry of the peak. For example, high peaks can be considered as pillars with spherical caps of radius $\xi / 2$. As we will see below for the description of the experiment [20] it is sufficient to use the simplest model for the peaks (flat faces).

At this point an important question is: with what precision can we calculate the roughness corrections? The term $-F^{\prime \prime \prime}(d) z^{3} / 3$ !, which is neglected in the Taylor expansion of $F(d-z)$, allows an estimation of the error in $\mathcal{F}_{P T}$. In the distance range that we are interested in here, $20<d<100 \mathrm{~nm}$, the force $F(d)$ behaves with the distance as $F(d)=A / d^{\alpha}$, where $A$ is a constant and $\alpha \approx 3.5$ [24]. Then the error is estimated as

$$
\Delta \mathcal{F}_{P T}=\gamma \frac{\alpha(\alpha+1)(\alpha+2)}{3 !}\left(\frac{w}{d}\right)^{3} F(d)
$$

where $\gamma$ is the skewness of the distribution $f(z)$. The data shown in fig. 1 give the largest $\gamma=1.285$ among the investigated films and we estimate the error as $\Delta \mathcal{F}_{P T} \approx$ $18.55(w / d)^{3} F(d)$. The latter means that the perturbation theory correction has meaning at least for $d / 4>w$. The minimal distance between rough surfaces $d_{0}$ depends on the area of nominal contact $L^{2}$, but even for $L$ as small as $1 \mu \mathrm{m}^{2}$ the condition $d_{0} / w>4$ is usually fulfilled [21. Therefore, we can now draw the important conclusion that the perturbation theory correction (10) has meaning up to the point of contact between interacting rough surfaces.

The precision with which we calculate the contribution of the high peaks is defined by the condition of applicability of the PFA to these peaks. This condition is $l\left(d_{1}\right) \gg d$ and we have for the error

$$
\Delta \mathcal{F}_{P F A}=(d / l) \mathcal{F}_{P F A}
$$

As we already mentioned we have to choose $d_{1}$ such that the condition $l\left(d_{1}\right) \gg d$ is true and, therefore, the correction (11) makes sense. Similarly, we can define the error $\Delta \mathcal{F}_{P F A}^{\prime}$ for the contribution of deep troughs (12). The relative error in (11) increases with the distance, but we have to keep in mind that $\mathcal{F}_{P F A}$ decreases very fast with $d$ and the absolute error stays small. The parameters $d_{1}$ and $d_{1}^{\prime}$ can be chosen rather arbitrarily if the conditions $l\left(d_{1}\right) \gg d$ and $l\left(d_{1}^{\prime}\right) \gg d$ are fulfilled. A practical recipe could be $d_{1}=\max \left[3 w,\left(d_{0}+w\right) / 2\right]$ and $d_{1}^{\prime}=\max \left[3 w,\left(d_{0}^{\prime}+w\right) / 2\right]$. It has to be noted that the contribution of deep troughs is always small, but we keep it for the sake of generality.

Results. - The roughness corrections (10)-(12) were deduced for the force between two rough parallel plates. In most of the experimental configurations the sphere-plate geometry is used. We can find the result for this configuration if the sphere's radius is large, $R \gg d$. This condition is typically true when the roughness effect is appreciable and we can apply the PFA to the total force $\mathcal{F}(d)$. The same equations (10)-(13) can be applied but now we have to understand $F(d)$ as the force between a smooth sphere and a smooth plate, approximated by

$$
F(d)=2 \pi R E(d) \quad R \gg d,
$$

where $E(d)$ is the Casimir-Lifshitz energy per unit area for the parallel plate configuration 9]. We neglect the thermal effect $(T=0)$ at short separations [12. However, we use measured optical properties of gold films [1] to account for the actual material properties.

We evaluated the force and all the roughness corrections to compare it with the experimental data 20]. The Lifshitz force $F(d)$ was calculated for the sphere radius $R=50 \mu \mathrm{m}$ using the optical data for sample 3 in 11 . The roughness effect was estimated for 800, 1200, and 1600 $\mathrm{nm} \mathrm{Au}$ films and a Au covered sphere. Here we present the results for the $1600 \mathrm{~nm}$ film. The roughness characteristics for combined sphere-plate AFM images were presented in ref. 21. For the $1600 \mathrm{~nm}$ film they are: rms roughness $w=10.1 \mathrm{~nm}$, the correlation length $\xi=42 \mathrm{~nm}$, and the distance upon contact $d_{0}=50.8 \pm 1.3 \mathrm{~nm}$. The last value was determined by electrostatic calibration [20. It is preferable to use this value, because $d_{0}$ determined from the roughness topography has a larger uncertainty [21. We used $d_{1}=\left(w+d_{0}\right) / 2=30.5 \mathrm{~nm}$. According to eq. (6) it corresponds to the average distance between high peaks, $l \approx 380 \mathrm{~nm}$. Note that the effective area of interaction is $L^{2}$, with $L=2100 \mathrm{~nm}$ [21]. For deep troughs the calculation details are less important. For the given $L$ we found $d_{0}^{\prime}=24.6 \mathrm{~nm}$. Since $d_{0}^{\prime}<3 w$ the troughs are not deep enough and can be taken into account perturbatively. Therefore, in this specific case there is no need to introduce $\mathcal{F}_{P F A}^{\prime}$.

The results are presented in fig. 2. One can see that the solid (blue) line, which shows the result of our approach, is in agreement with the experimental data within the experimental errors. This is in contrast with the perturbation theory approach that failed to explain the data [20]. This is demonstrated in the inset, which shows different components of this force. At short distances the contribution of high peaks (2, red) is so large that it dominates the whole force. In this case a few peaks become very close 


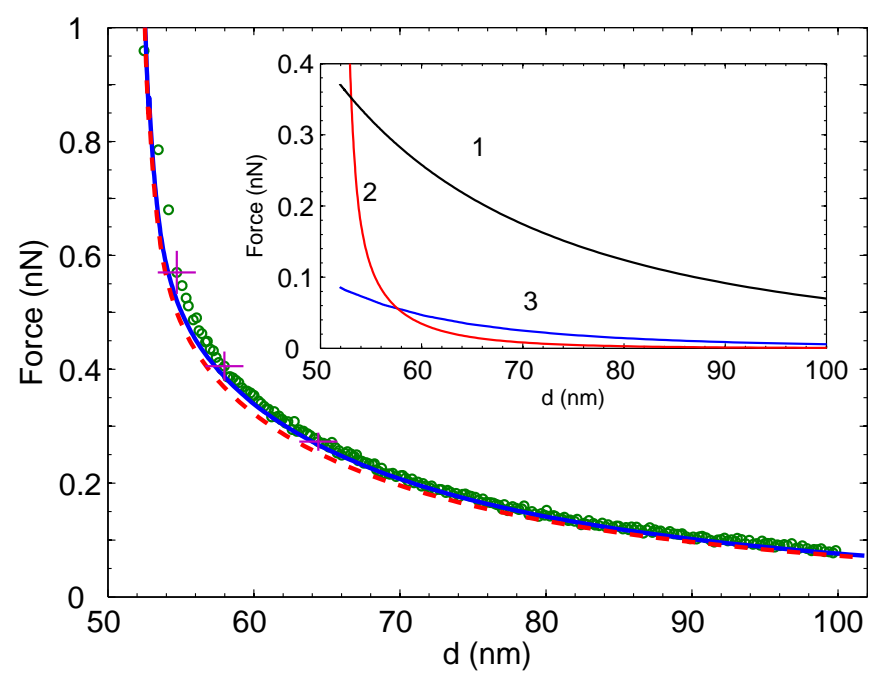

Fig. 2: (Color online) The force between a Au covered sphere $(R=50 \mu \mathrm{m})$ and a plate $(1600 \mathrm{~nm}$ thick $\mathrm{Au})$. The open (green) circles are the experimental data from [20. The vertical and horizontal bars show the experimental errors for a few points. The solid (blue) line is the result of our model. Naive application of the PFA to the force between rough bodies based on eqs. (8) and (16) is shown by the dashed (red) curve. The inset shows different components of the force. 1 (black) is the force $F(d)$ between smooth surfaces calculated according to the Lifshitz formula. 2 (red) is the contribution of the high peaks according to eq. (11). 3 (blue) is the perturbation theory correction according to eq. (10). The sum of all three curves gives the solid line in the main panel.

to the opposite body so that the force diverges at $d \rightarrow d_{0}$. There can be very few high peaks but their contribution cannot be neglected. On the other hand the contribution of high peaks disappears very fast when the distance becomes larger.

We used two different models to calculate the contribution of high peaks in eq. (11). In the first model the peak was considered as a pillar with a flat face. In the second model the peak had a spherical cap of radius $\xi / 2$. The interaction of the cap with a plate was taken into account according to ref. [25], where the proximity force approximation is not used. We found a negligible difference between the two models of peaks. The reason is the following: When the distance $d-d_{0} \sim \xi$, then the contribution of the peaks is very small due to their small area of interaction. When $d$ approaches $d_{0}$ or $d-d_{0} \ll \xi$, the contribution of high peaks becomes significant, but the shape of the peaks is not important anymore, because the PFA is valid in this limit.

Naive application of the proximity force approximation according to eqs. (8) and (16) gives the dashed line (red) in fig. 2 It is interesting to note that this line is also in agreement with the experimental data. At the shortest separations both curves coincide, because the dominating high peaks can be treated with the PFA. At larger distances the perturbative contribution becomes important and the PFA result lies below the solid line as it should be [17. However, the difference between these two curves is within the experimental errors. Perturbation theory accounts for the non-additivity of the Casimir force, whereas the PFA assumes it is additive. So this difference provides an indication of the effect of the non-additivity in the roughness correction. It can be concluded that within the experimental error the experiment in ref. [20] was not sensitive to this non-additivity.

Conclusions. - In conclusion, we developed a reliable method to include the effect of roughness of interacting bodies in the Casimir force at short distances when perturbation theory fails. It was established that roughness of gold films can be described asymptotically (for high peaks or deep troughs) by extreme value statistics. In this case the rough surface can be presented as a large number of asperities with heights of the order of the rms roughness and a few occasional peaks, which are much higher than the rms roughness. The distance between high peaks is large so that one can calculate their contribution for each peak separately (using the PFA). The smaller asperities can be calculated using perturbation theory beyond the PFA. The contribution of high peaks is extremely important for short separations, where it dominates not only the perturbative roughness correction but also the force as a whole. Therefore, our result is interesting not only for the Casimir force but also for the problem of adhesion between surfaces in general 26, including wet environments 27. 29.

We repeat that the method presented here solves the significant discrepancy between measurements of the Casimir force at short separations, and the results of perturbation theory 20.

\section{$* * *$}

The authors benefited from exchange of ideas within the ESF Research Network CASIMIR. We would also like to thank P. J. van Zwol and B. J. Hoenders for useful discussions.

\section{REFERENCES}

[1] H. B. G. Casimir, Proc. K. Ned. Akad. Wet. 51, 793 (1948).

[2] F. Capasso, J. N. Munday, D. Iannuzzi, and H. B. Chan, IEEE J. Sel. Top. Quantum Electron. 13, 400 (2007).

[3] A. W. Rodriguez, F. Capasso, and S. G. Johnson, Nature Photon. 5, 11 (2011).

[4] P. Ball, Nature 447, 772 (2007).

[5] F. M. Serry, D. Walliser, and G. J. Maclay, J. Appl. Phys. 84, 2501 (1998).

[6] E. Buks and M. L. Roukes, Europhys. Lett. 54, 220 (2001).

[7] E. Buks and M. L. Roukes, Phys. Rev. B 63, 033402 (2001).

[8] I. E. Dzyaloshinskii, E. M. Lifshitz and L. P. Pitaevskii, Advances in Physics 38, 165 (1961). 
[9] E. M. Lifshitz and L. P. Pitaevskii, Statistical Physics (Pergamon Press, Oxford, 1980) Pt. 2.

[10] A. Lambrecht and S. Reynaud, Eur. Phys. J. D 8, 309 (2000).

[11] V. B. Svetovoy, P. J. van Zwol, G. Palasantzas, and J. T. M. De Hosson, Phys. Rev. B 77, 035439 (2008).

[12] K. A. Milton, J. Phys. A: Math. Gen. 37, R209 (2004).

[13] A. O. Sushkov, W. J. Kim, D. A. R. Dalvit, and S. K. Lamoreaux, Nature Phys. 7, 230 (2011).

[14] G. L. Klimchitskaya and Yu. V. Pavlov, Int. J. Mod. Phys. A 11, 3723 (1996).

[15] B. V. Derjaguin, Kolloid Z. 69, 155 (1934).

[16] B. V. Derjaguin, I. I. Abrikosova, and E. M. Lifshitz, Quart. Rev. Chem. Soc. 10, 295 (1956).

[17] C. Genet, A. Lambrecht, P. Maia Neto and S. Reynaud, Europhys. Lett. 62, 484 (2003).

[18] P. A. Maia Neto, A. Lambrecht, and S. Reynaud, Europhys. Lett. 69, 924 (2005).

[19] P. A. Maia Neto, A. Lambrecht, and S. Reynaud, Phys. Rev. A 72, 012115 (2005).

[20] P. J. van Zwol, G. Palasantzas, and J. Th. M. De Hosson, Phys. Rev. B 77, 075412 (2008).

[21] P. J. van Zwol, V. B. Svetovoy, and G. Palasantzas, Phys. Rev. B 80, 235401 (2009).

[22] S. Coles, An Introduction to Statistical Modelling of Extreme Values (Springer, Berlin, 2001).

[23] E. J. Gumbel, Statistics of Extremes (Dover Publications Inc., 2004).

[24] G. Palasantzas, V. B. Svetovoy, and P. J. van Zwol, Int. J. Mod. Phys. B 24, 6013 (2010).

[25] A. Canaguier-Durand, P. A. M. Neto, I. Cavero-Pelaez, A. Lambrecht, and S. Reynaud, Phys. Rev. Lett. 102, 230404 (2009).

[26] F. W. DelRio, M. P. de Boer, J. A. Knapp, E. D. Reedy, Jr., P. J. Clews, and M. L. Dunn, Nature Mater. 4, 629 (2005).

[27] F. W. DelRio, M. L. Dunn, L. M. Phinney, and C. J. Bourdon, Appl. Phys. Lett. 90, 163104 (2007).

[28] P. J. van Zwol, G. Palasantzas, and J. T. M. De Hosson, Phys. Rev. E 78, 031606 (2008).

[29] B. N. J. Persson, J. Phys.: Condens. Matter 20, 315007 (2008). 